\title{
Behavior and Outcomes of Pregnancy Associated Breast Cancer
}

\author{
Kausar Suleman ${ }^{1 *}$, Asif Husain Osmani ${ }^{1}$, Hashem Al Hashem ${ }^{1}$, Taher Al \\ Twegieri $^{1}$, Dahish Ajarim ${ }^{1}$, Noha Jastaniyah ${ }^{2}$, Wafa Al Khayal ${ }^{3}$, Osama Al Malik ${ }^{3}$, \\ Adhar Al Sayed ${ }^{1}$
}

\begin{abstract}
Introduction: Pregnancy Associated Breast cancer (PABC) is associated with poor prognosis and a decreased overall survival. A retrospective review was conducted to review the experience and outcome in a tertiary care hospital, and to compare those seen in a matched group for year of diagnosis. Materials and Methods: This is a retrospective review of a prospectively collected breast cancer registry. The study was conducted in a tertiary care hospital in Riyadh, Saudi Arabia from January to Decamber 2014 . Female patients with PABC were identified and matched with similar cohort of non-pregnant breast cancer patients that were diagnosed between 2001-2010. Clinical data including age, tumor biology, clinical stage, follow up and outcomes (disease free survival, DFS) were analyzed and compared between the two groups using SAS 9.3 and R-2.14.1 Results: A total of 110 patients in Group 1 and 114 patients in Group II were analyzed. In both groups, the patient age ranged was between 20 to 45 years; the median follow up was 34 months in PABC and 54 months in non-pregnant cohort. PABC were statistically more likely to be triple negative ( $\mathrm{p}$ value- 0.05$)$ and diagnosed at advanced stage (stage 3 and 4) (p value-0.02). There was no difference in the occurrence of Her- 2 positive disease. In pregnant patients there was a 5-year survival rate of $65 \%$ compared to non-pregnant cohort of $82 \%$ with $p$ value of 0.002 and DFS was also $47.5 \%$ versus $65.4 \%$ with a p value .002 which is statistically significant. Conclusion: Pregnancy associated breast cancer (PABC) is diagnosed at a more advanced stage and tends to be triple negative and they are associated with a worse DFS and overall survival. Early detection during pregnancy may improve outcome.
\end{abstract}

Keywords: Breast cancer (BC)- pregnancy- prognosis- pregnancy associated breast cancer (PABC)

Asian Pac J Cancer Prev, 20 (1), 135-138

\section{Introduction}

Pregnancy associated breast cancer (PABC) is a relatively rare clinical presentation. It's defined as breast cancer diagnosed during pregnancy or one-year following delivery (Psyrri, 2005). In developed countries he incidence of PABC during pregnancies is estimated between $1 / 10,000$ to $1 / 3,000$. In previous studies, the rate of PABC in women younger than 45 years of age varied from $2.6 \%$ to $7 \%$ (Andersson et al., 2015; Vinatier et al., 2009). The incidence is currently increasing as more women are delaying their childbearing in industrialized nations (Andersson et al., 2015). In a large cohort population study of more than sixteen thousand patients aged between 15 to 44 years of age, PABC represents $7 \%(1,161$ cases $)$ of all breast cancers in this cohort, which doubled in incidence, from 16.0 to $37.4 / 100,000$ childbirths during the study period (1963 to 2002) (Andersson et al., 2009). In this population, 30.1\% of PABC patients were young between the ages of 25 and 29 year (Andersson et al., 2009).
The prognosis of PABC has been discussed in multiple studies with inconsistent results (Stensheim et al., 2009; Halaska et al., 2009). Amant et al., (2013) conducted an international collaborative study, a total of 311 PABC were eligible for analysis and they found there was no significant difference in both disease free survival and overall survival. While, in a met-analysis of 30 studies looking at PABC found that it was associated with poorer prognosis especially if diagnosed immediately post-partum (Azim et al., 2012). Breast cancer is an important health problem in Saudi Arabia. It ranks first among all newly diagnosed malignant diseases in Saudi Arabia and among women, accounting for $29.1 \%$ in the year 2013. It was reported that the incidence of breast cancer in patients less than 44 years of age was reported as $48.8 \%$ (Alghamdi et al., 2013). In Saudi Arabia, the average pregnancy rate is high, and women are likely to develop breast cancer at a younger age, it is plausible that a high frequency of PABC is to be expected (Ibrahim et al., 2000) Hence, association of pregnancy with Breast cancer frequently imposes serious management implications. 
In this paper we aim to compare patients with $\mathrm{PABC}$ with a similar cohort of non PABC Patients, taking into regard the year of diagnosis for their outcomes.

\section{Materials and Methods}

This is a retrospective cohort review. The study was conducted in King Faisal Specialist hospital and Research Centre, Riyadh, Saudi Arabia; institutional review board (IRB) approval was obtained prior to starting the project. The patient's files and the breast cancer registry between the years 2001 to 2010 were reviewed and a total of 224 eligible individuals were identified. The study was conducted in a tertiary care hospital in Riyadh, Saudi Arabia from January to Decamber 2014. Pregnancy associated breast cancer (PABC) was defined in the inclusion criteria as patients diagnosed with breast cancer while pregnant. A total of 110 female PABC was identified and a further, 114 female non-pregnant breast cancer patients diagnosed in the same time frame during the period of 2001 to 2010 were identified and included as a comparison.

Stage 1 and 2 patients were classified as Early Stage, Stage 3 and 4 patients were classified as Advanced Stage patients. Her- 2 was considered positive if it was +3 or if it was +2 and the FISH amplified. Statistical analysis is intended to test the hypothesis that pregnant breast cancer women have different outcomes in comparison to the non-pregnant breast cancer women. Statistical analysis was conducted using SAS 9.3 and R-2.14.1. Overall Survival (OS) probabilities have been calculated using Kaplan-Meier method. Log-Rank test has been used to compare the difference between the survival probabilities. Cox regression model was used to obtain difference in hazard rates between two groups.

\section{Results}

A total of 110 patients in PABC group 1 and 114 patients in non-pregnant group were identified during the study period. In PABC, the median age was 34 years (20 -45 years) whereas in non-pregnant group was 44 years (24 to 48 years), all the patients were diagnosed at the same time interval. The median follow up was 34 months in PABC group and 54 months in non-pregnant group. PABC consisted of more triple negative disease $(23 \%)$ compared to $15 \%$ non-pregnant group and this was found to be statistically different ( $\mathrm{P}$ value=0.05). In the PABC group $55.7 \%$ presented at an advanced stage while only $36.7 \%$ of the non-pregnant group presented late, this was also statistically significant $(\mathrm{P}$ value $=0.01 \%)($ Table 1$)$. Her-2 positive and negative patients were fairly the same in both groups. In pregnant patients, 33 patients have been died while 84 patients were alive. The 5 -year survival rate was $65 \%$. However, in non-pregnant patients, 20 have

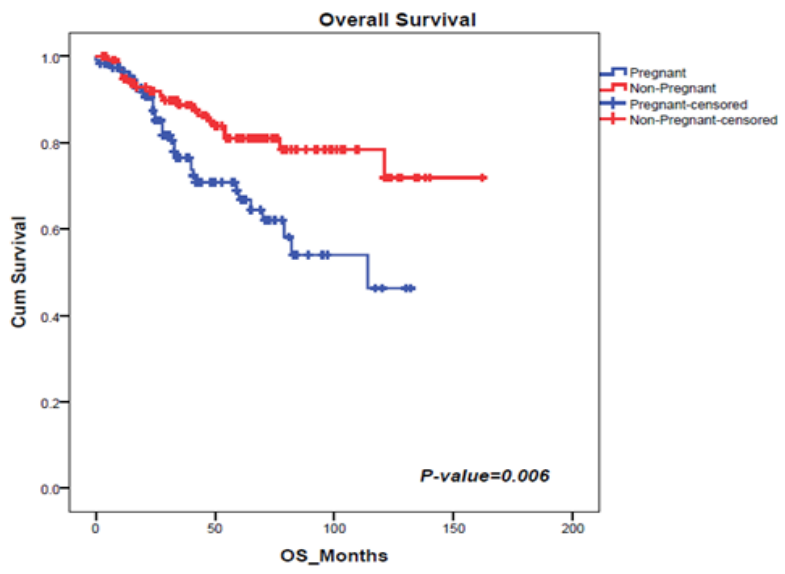

Figure 1. Kaplan-Meier Curve of the Cumulative Survival and Overall Survival in Months. The $P$-value is derived from log rank test for overall comparison of overall survival.

Table 1. Baseline Characteristics and Outcomes

\begin{tabular}{|c|c|c|c|c|c|c|c|}
\hline \multirow[b]{2}{*}{ Median Age years } & \multicolumn{2}{|c|}{ Pregnant } & \multicolumn{4}{|c|}{ Not-Pregnant } & \multirow[b]{2}{*}{ P-value } \\
\hline & $34(20$ to 45$)$ & & $95 \% \mathrm{CI}$ & $44(24$ to 48$)$ & & $95 \% \mathrm{CI}$ & \\
\hline $\mathrm{ER}+/ \mathrm{PR}+$ & 43 & $35.80 \%$ & & 58 & $48.70 \%$ & & 0.2 \\
\hline $\mathrm{ER}+/ \mathrm{PR}-$ & 8 & $6.70 \%$ & & 10 & $8.40 \%$ & & \\
\hline ER-/PR+ & 7 & $5.80 \%$ & & 5 & $4.20 \%$ & & \\
\hline ER-/PR- & 57 & $47.50 \%$ & & 43 & $36 \%$ & & \\
\hline Un Known & 5 & $4.00 \%$ & & 3 & $3 \%$ & & \\
\hline Triple negative & 29 & $24.00 \%$ & & 17 & $14 \%$ & & 0.05 \\
\hline non-triple -ve & 93 & $76.00 \%$ & & 103 & $85.80 \%$ & & \\
\hline Early Stage & 42 & $34.00 \%$ & & 59 & $49 \%$ & & 0.01 \\
\hline Advanced-stage & 68 & $55.70 \%$ & & 44 & $36.70 \%$ & & \\
\hline Not Known & 12 & $10.00 \%$ & & 17 & $14 \%$ & & \\
\hline Median follow-up (months) & $34(1$ to 132$)$ & & & $54(3$ to 162$)$ & & & \\
\hline Her 2 -ve status & 63 & $60.6 \%$ & & 57 & $61 \%$ & & 0.5 \\
\hline Her $2+$ ve status & 41 & $39.40 \%$ & & 36 & $38.70 \%$ & & \\
\hline OS & 117 & $68.90 \%$ & 0.05 & 120 & $81 \%$ & 0.04 & 0.006 \\
\hline DFS & 117 & $50.80 \%$ & 0.05 & 120 & $64 \%$ & 0.05 & 0.01 \\
\hline
\end{tabular}




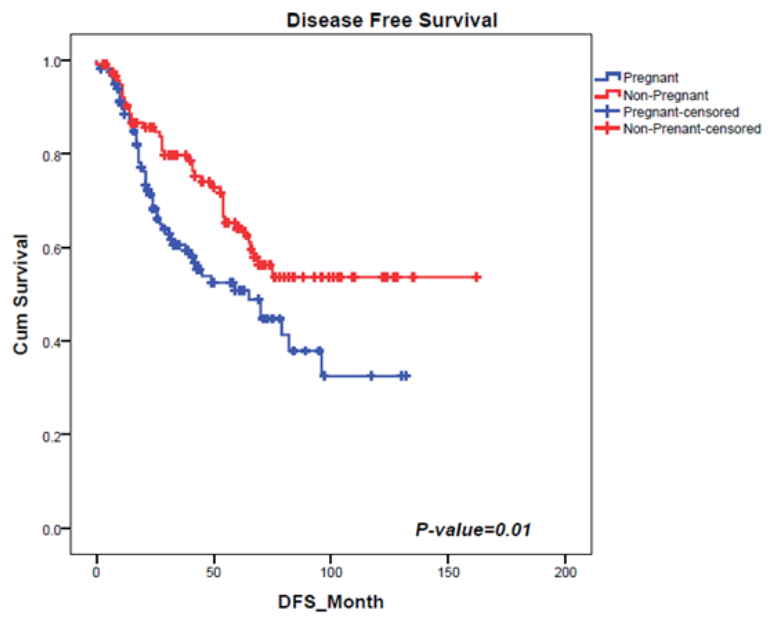

Figure 2. Kaplan-Meier Curve of the Cumulative Survival and Disease Free Survival in Months. The $P$-value is derived from $\log$ rank test for overall comparison of disease free

been denied while 100 were censored. The survivial propability was $82 \%$, (P value $=0.002)($ Figure 1$)$. Disease free survival (DFS) was $47.5 \%$ vs $65.4 \%$ (P value $=0.002$ ). (Figure 2). thirty-three patients have been relapsed or died in remission, while 75 patients were censored.

\section{Discussion}

In this retrospective review study amongst women of childbearing age, we observed that the median age of PABC patients was more trending towards younger age from their matched controls, even in selected young population. Furthermore, they were diagnosed at a more advanced stage and had a poorer immuno-histological prognostic profile, with more tumors being negative for hormonal receptors, both ER and PR and Her 2/neu negative. Our results showed that patients presented with advance stage with PABCs and this is described frequently in PABC (Beadle et al., 2009; Stensheim et al., 2009; Rodriguez et al., 2008). Furthermore, according to Genin et al. in comparison with the non-PABCs, PABC group has a greater HER2 expression and hormone receptor (ER, PR) negative status which adds to their unfavorable prognosis and were more likely to present with T3 or T4 tumors (Genin et al., 2012). Some mechanisms may potentially explain the association between pregnancy and a worse outcome. The changes that occur in the breast during pregnancy is well-known, one study compared surgical specimens of PABC patients and non-pregnant controls and it was seen that there was a significant increase in angiogenesis in tumor tissues and healthy breast tissue in the PABC when compared with the non-pregnant controls, however, there was no difference seen in lymphangiogeneis (Genin et al., 2014). Most PABCs do not express hormone receptors, one theory studied observed that high levels of circulation estrogen promoted the formation of ER-negative cancers and it also increased systemic angiogenesis, stromalization and bone marrow cell recruitment (Gupta et al., 2007).

Our results are also similar with other studies that showed PABC was associated with poor prognosis, shorter 5-year survival rate and DFS against its matched controls. Maggard et al looked at specifically outcomes of young patients with breast cancer reviewed 4,616 young females using the Surveillance, Epidemiology, and End Results cancer database described that young patients had worse 5 year survival and tended to present with advanced stage of disease and have more estrogen and progesterone negative tumors (Maggard et al., 2003). A recent metanalysis of 41 studies that looked that prognosis of PABC found that breast cancer diagnosed during pregnancy was more likely to result in death and decreased disease free survival (Hartman, 2016 ).

In Saudi Arabia, it was reported in the Cancer registry that $42 \%$ were diagnosed with lymph node spread (regional) and 13\% had distant metastasis at the time of diagnosis and these stages have a lower survival advantage than early stage breast cancer. Earlier studies conducted in different regions of Saudi Arabia such as Buraidah, Riyadh, and Al Khobar, have explored female knowledge, and the attitude towards breast cancer, which revealed lack of knowledge about the risk factors for breast cancer; and lack of understanding of the importance of self-breast examination and screening mammography (Alam, 2006; Dandash and Al-Mohaimeed, 2007; Amin et al., 2009 ). These factors hinder the diagnosis of breast cancer at an early stage in both pregnant patients and non-pregnant patients. Other, factors in pregnancy and breast feeding that can further delay diagnosis is that increase in the breast density makes clinical examinations and breast imaging more difficult to interpret.

According to Azim et al., (2012) met analysis, the prognosis of breast cancer arising during pregnancy was significantly associated with poor overall survival, based on the pooled analyses of the univariate and multivariate models. Another case matched control study that matched patients for age, year of surgery, stage and neoadjuvant chemotherapy found that there was no difference in tumor characteristics between the two groups; however, they found that PABC had significantly poorer DFS and a trend of a worse OS.

In conclusion, patients diagnosed with $\mathrm{PABC}$ are independently associated with more advanced stage, poor immune-histochemistry leading to poor survival. PABC should be recognized as a special category of breast cancer that is associated with poor outcomes. The majority of the population in Saudi Arabia and most developing countries are younger and breast cancer is frequently diagnosed in these childbearing years. This leads us to suggest that awareness programs for both the population and the healthcare professional about PABC would lead to early detection and prompt management thereby leading to better outcomes. Furthermore, research of the tumor biology beyond phenotyping is warranted as this will lead to better understanding of PABC and guide treatment.

\section{Competing interests}

The authors declare that there are no competing interests. This study was approved by institutional ethical comitee ONC RU/200/35. We would like acknowledge our breast oncology group for their support. This study required no funding.

Asian Pacific Journal of Cancer Prevention, Vol 20 


\section{References}

Alam AA (2006). Knowledge of breast cancer and its risk and protective factors among women in Riyadh. Ann Saudi Med, 26, 272-7.

Alghamdi IG (2013). The incidence rate of female breast cancer in Saudi Arabia: an observational descriptive epidemiological analysis of data from Saudi Cancer Registry 2001-2008. Breast Cancer, 5, 103-9

Amant F (2013). Prognosis of women with primary breast cancer diagnosed during pregnancy: Results from an international collaborative study. J Clin Oncol, 31, 2532-39.

Amin TT, Al Mulhim AR, Al Meqihwi A (2009). Breast cancer knowledge, risk factors and screening among adult Saudi women in a primary health care setting. Asian Pac J Cancer Prev, 10, 133-8.

Andersson TM (2009). Increasing incidence of pregnancyassociated breast cancer in Sweden. Obstet Gynecol, 114, $568-72$.

Andersson TM ( 2015). Cancer during pregnancy and the postpartum period: A population-based study. Cancer, 121, 2072-7.

Azim HA Jr (2012). Prognosis of pregnancy-associated breast cancer: a meta-analysis of 30 studies. Cancer Treat Rev, 38, 834-42.

Azim HA Jr (2012). The biological features and prognosis of breast cancer diagnosed during pregnancy: A case-control study. Acta Oncologica, 51, 653-61.

Beadle BM, Woodward WA, Middleton LP, et al (2009). The impact of pregnancy on breast cancer outcomes in women $<$ or $=35$ years. Cancer, 115, 1174-84.

Dandash KF, Al-Mohaimeed A (2007). Knowledge, attitudes, and practices surrounding breast cancer and screening in female teachers of Buraidah, Saudi Arabia. Int $J$ Health Sci, 1, 61-71.

Genin AS (2012). Pregnancy-associated breast cancers: do they differ from other breast cancers in young women? Breast $J$, 21, 550-5.

Genin AS (2014). Pregnancy stimulates tumor angiogenesis in breast carcinoma. Anticancer Res, 34, 125-31.

Gupta PB (2007). Systemic stromal effects of estrogen promote the growth of estrogen receptor-negative cancers. Cancer Res, 67, 2062.

Halaska MJ (2009). Presentation, management and outcome of 32 patients with pregnancy-associated breast cancer: a matched controlled study. Breast $J, \mathbf{1 5}, 461-7$.

Hartman EK, Eslick GD (2016). The prognosis of women diagnosed with breast cancer before, during and after pregnancy: a meta-analysis. Breast Cancer Res Treat, 160, $347-60$.

Ibrahim E (2000). Pregnancy-associated breast cancer: a case-control study in a young population with a high-fertility rate. Med Oncol, 17, 293-300.

Maggard MA (2003). Do young breast cancer patients have worse outcomes?. J Surg Res, 113, 109-13.

Psyrri A, Burtness B (2005). Pregnancy-associated breast cancer. Cancer J, 11, 83-95.

Rodriguez AO (2008). Evidence of poorer survival in pregnancy-associated breast cancer. Obstet Gynecol, 112, 71- 8 .

Stensheim H (2009). Cause-specific survival for women diagnosed with cancer during pregnancy or lactation: a registry-based cohort study. J Clin Oncol, 27, 45-51.

Vinatier E, Merlot B, Poncelet E, Collinet P, Vinatier D (2009). Breast cancer during pregnancy. Eur J Obstet Gynecol Reprod Biol, 147, 9-14.
This work is licensed under a Creative Commons AttributionNon Commercial 4.0 International License. 